\title{
Performance Comparison of Energy Efficient Protocols for Wireless Sensor Networks (WSN)
}

\author{
Pooja Singh \\ Deptt.of Computer Science, \\ Amity School of Engg. \& Tech \\ GSSIPU, New Delhi, India
}

\author{
Vikas Pareek, Ph.D \\ Deptt. Of Computer Science, \\ Banasthali Vidhyapith Univ. \\ Jaipur, Rajsthan, India
}

\author{
Anil k Ahlawat, Ph.D \\ Deptt. Of MCA, KIET-UPTU \\ Ghaziabad, India
}

\begin{abstract}
Wireless sensor networks have recently gained a lot of attention from the researches. Wireless sensor networks are often partitioned into clusters, each managed by a cluster head (gateway). This paper reviews medium access control (MAC), an enabling technology in wireless sensor networks. MAC protocols control how sensors access a shared radio channel to communicate with neighbors in small area coverage. It classifies traditional (IEEE 802.11) and existing MAC protocols and a power efficient gathering protocol, known as PEGASIS, as examples of MAC protocol designed specifically for a sensor network. SENSOR MAC sets the radio to sleep during transmissions of other nodes unlike PEGASIS which involves non sleeping cycles. Finally this paper compares the performance of both protocols and displays the results.
\end{abstract}

\section{General Terms}

Small Sensor Network, Sensor - MAC, PEGASIS

\section{Keywords}

S - MAC, WSN, IEEE 802.11, Fuse Data, Energy, Node.

\section{INTRODUCTION}

Wireless Sensor Networks (WSN) are collaborative network of small wireless sensor devices, sensing a physical phenomenon and then the information gathered is processed to get relevant result. In the other way, these network consists of a large number of battery-powered sensors capable of communicating wireless [23]. They are distributed within an area of interest in order to track, measure and monitor various events. Wireless sensor networking is an emerging technology that has a wide range of potential applications including environment monitoring, smart spaces, medical systems and robotic exploration. Protocols for these networks need to be extremely adaptable and scalable because of constant changes in network topology. Wireless sensor network actually multihop / mesh network proto-type. Medium Access Control (MAC) is an important technique [2,6], that enables the successful operation of the network. One fundamental task of the MAC protocol is to avoid collisions so that two interfering nodes do not transmit at the same time. If high energy efficiency demands are also considered, it becomes clear that the design of MAC protocols for WSN is a difficult task. To design a good MAC protocol for the wireless sensor networks, we have considered the following attributes. The first is the throughput then packet delay fraction and lastly end to end delay. As stated before Sensor nodes are likely to be battery powered, and it is often very difficult to change or recharge batteries for these nodes. In fact, someday there will be expectation that some nodes to be cheap enough that they are discarded rather than recharged. Prolonging network lifetime for these nodes is a critical issue. Another important attribute is scalability and adaptively to changes in network size, node density and topology. Some nodes may die over time; some new nodes may join later, some nodes may move to different locations. A good MAC protocol should gracefully accommodate such network behavior changes. MAC protocol explicitly designed for wireless sensor networks and Power Efficient Gathering in Sensor Information Systems (PEGASIS) based on traditional IEEE 802.11 MAC. The major source of inefficiency is idle listening, i.e., listening to receive possible traffic that is not sent. If nothing is sensed, nodes are in idle mode for most of the time. However, in many MAC protocols such as IEEE 802.11 ad hoc mode or CDMA nodes have to listen to the channel to receive possible traffic. Sensor-MAC reduces idle listening by periodically putting nodes into sleep state. In protocols for traditional data networks like the IEEE 802.11, bandwidth utilization is a big concern, and nodes normally operate in fully active mode. In SENSOR MAC (S-MAC), however, the nodes only become more active when there is traffic in the network. To reduce the control overhead and latency, SENSOR MAC introduces coordinated sleeping among neighboring nodes. Based on traditional IEEE 802.11 MAC, PEGASIS (Power-Efficient Gathering in Sensor Information Systems) [19], which is near to optimal for that type of data gathering application in sensor networks, can nodes data move intelligently. The key idea in PEGASIS [22, 19, and 9] is to form a chain among the sensor nodes so that each node will receive from and transmit to a close neighbor. Gathered data moves from node to node, get fused, and eventually a designated node transmits to the BS. Nodes take turns transmitting to the BS so that the average energy spent by each node per round is reduced. Building a chain to minimize the total length is similar to the traveling salesman problem, which is known to be intractable. However, with the radio communication energy parameters, a simple chain built with an approach performs quite well. If nodes are not within transmission range of each other, then alternative, possibly multi-hop transmission paths will have to be used. Since PEGASIS believes on chain based schemes which will not be affected that much as each node communicates only with a local neighbor and we can use a multi-hop path to transmit to the BS. We need to make some adjustments in the chain construction procedure to ensure that no node is left out. The Sensor-MAC protocol relies on direct reachability to function correctly. To ensure balanced energy dissipation in the network, an additional parameter could be considered to compensate for nodes that must do more work every round. If the sensor nodes have different initial energy levels, then we could consider the remaining energy level for each node in addition to the energy cost of the transmissions. The assumption of location information is not critical. The BS can determine the locations and transmit to all nodes, or the 
nodes can determine this through received signal strengths. For example, nodes could transmit progressively reduced signal strengths to find a close neighbor to exchange data. This would require the nodes to consume some energy when trying to find local neighbors, however, this is only a fixed initial energy cost when constructing the chain. If nodes are mobile, then different methods of transmission could be examined. For instance, if nodes could approximate how often and at what speed other nodes are moving, then it could determine more intelligently how much power is needed to reach the other nodes. Perhaps, the BS can help coordinate the activities of nodes in data transmissions.

For example, The Radio Model for PEGASIS:

Here take the radio model as reference [3, 19] which is the first order radio model. In this model, a radio dissipates with

Eelec $=50 \mathrm{~nJ} / \mathrm{bit}$ to run the transmitter or receiver circuitry and

Îamp $=100 \mathrm{pJ} / \mathrm{bit} / \mathrm{m} 2$; for the transmitter amplifier.

The radios have power control and can expend the minimum required energy to reach the intended recipients. The radios can be turned off to avoid receiving unintended transmissions. An r2 energy loss is used due to channel transmission [8, 11]. The equations used to calculate transmission costs and receiving costs for a k-bit message and a distance $\mathrm{d}$ are shown below:

\section{Transmitting}

ETx $(k, d)=E T x-$ elec $(k)+E T x-a m p(k, d)$

$\operatorname{ETx}(\mathrm{k}, \mathrm{d})=$ Eelec $* \mathrm{k}+$ Îamp $* \mathrm{k} * \mathrm{~d} 2$

Receiving

$\operatorname{ERx}(\mathrm{k})=\mathrm{ERx}-\mathrm{elec}(\mathrm{k})$

$\operatorname{ERx}(\mathrm{k})=$ Eelec $* \mathrm{k}$

Receiving is also a high cost operation, therefore, the number of receives and transmissions should be minimal.

\section{RELATED WORK}

Medium access control is a broad research area, including work in the new area of low power and wireless sensor networks. The reasons for the unnecessary energy waste in wireless communication are:

- Packet collision: It can occur when nodes don't listen to the medium before transmitting.

- Overhearing: A node receives a packet which is addressed to another node.

- Idle listening: The main reason for energy waste is when a node listens to an idle channel waiting to receive data.

In order to satisfy WSN needs, the MAC protocols have to fulfil the following requirements:

- Energy efficiency: Lifetime is possible by designing energy-efficient protocols.

- Collision avoidance: The main goal is to reduce collisions as much as possible.

- Scalability and adaptability: The MAC protocol needs to be adaptable to changes in network topology

- Latency: Latency represents the delay of a packet when sent through the network.
- Throughput: Represents the amount of data within a period of time sent from sender to the receiver through WSN.

The design for wireless sensor networks can be broadly divided into contention-based and TDMA protocols.

\section{DESCRIPTION OF MAC LAYER PROTOCOL}

\subsection{Sensor - MAC}

The main idea of SENSOR MAC is to put nodes to sleep from time to time to reduce energy wastage caused by the above events. A node goes to sleep periodically if it is not engaged in transmission or reception, to reduce idle listening. It also goes to sleep if its neighbours are involved in communication, of which it is not a party, to reduce collision and overhearing.

A cycle in SENSOR MAC consists of a listen and a sleep state. A node normally follows predetermined schedules to wake up or go to sleep $[9,12]$, with the following exceptions:

(i) A node goes to sleep if any of its neighbours are communicating, and the node is not a party.

(ii) A node wakes up at the end of its neighbour's transmission if it needs to relay the packet.

This is done by overhearing neighbour's [12, 14, 23] RTS and CTS exchanges before the node goes to sleep and serves the purpose of reducing latency caused by sleeping. This behaviour is called adaptive listening. Schedules are periodically exchanged by broadcasting SYNC packets among neighbouring nodes to induce synchronized listen behaviour as much as possible and thus to reduce latency caused by sleeping. Synchronized neighbours form a virtual cluster, but synchronization can only be achieved to a certain extent in an ad hoc environment. Lack of complete synchronization introduces sleep delay which results in increased packet latency. However, SENSOR MAC has a fixed contention window, as a change in contention window size changes the length of an active period and hence influences the synchronization process. Moreover, when a node fails to win the contention or it encounters an RTS collision, it goes to sleep until the next active period. On the other hand, when a node sends out an RTS successfully, it does not go back to sleep until the transmitted DATA packet is acknowledged.

Scheduling: The first technique in SENSOR MAC, fig 1, is to establish low duty-cycle operation on nodes in a multi-hop network. For long-lived sensor networks, we expect duty cycles of $1-10 \%$. The basic scheme is similar to the 802.11 PS modes, but without assuming all nodes can hear each other, or a designated base-station. Data transmission: The collision avoidance mechanism in SENSOR MAC is similar to that in the IEEE 802.11. Contention only happens at a receiver's listen interval. SENSOR MAC uses both virtual and physical carrier sense. Unicast packets combine CSMA with an RTS-CTS-DA T A-ACK exchange be-tween the sender and the receiver, while broadcast packet use only CSMA procedure 

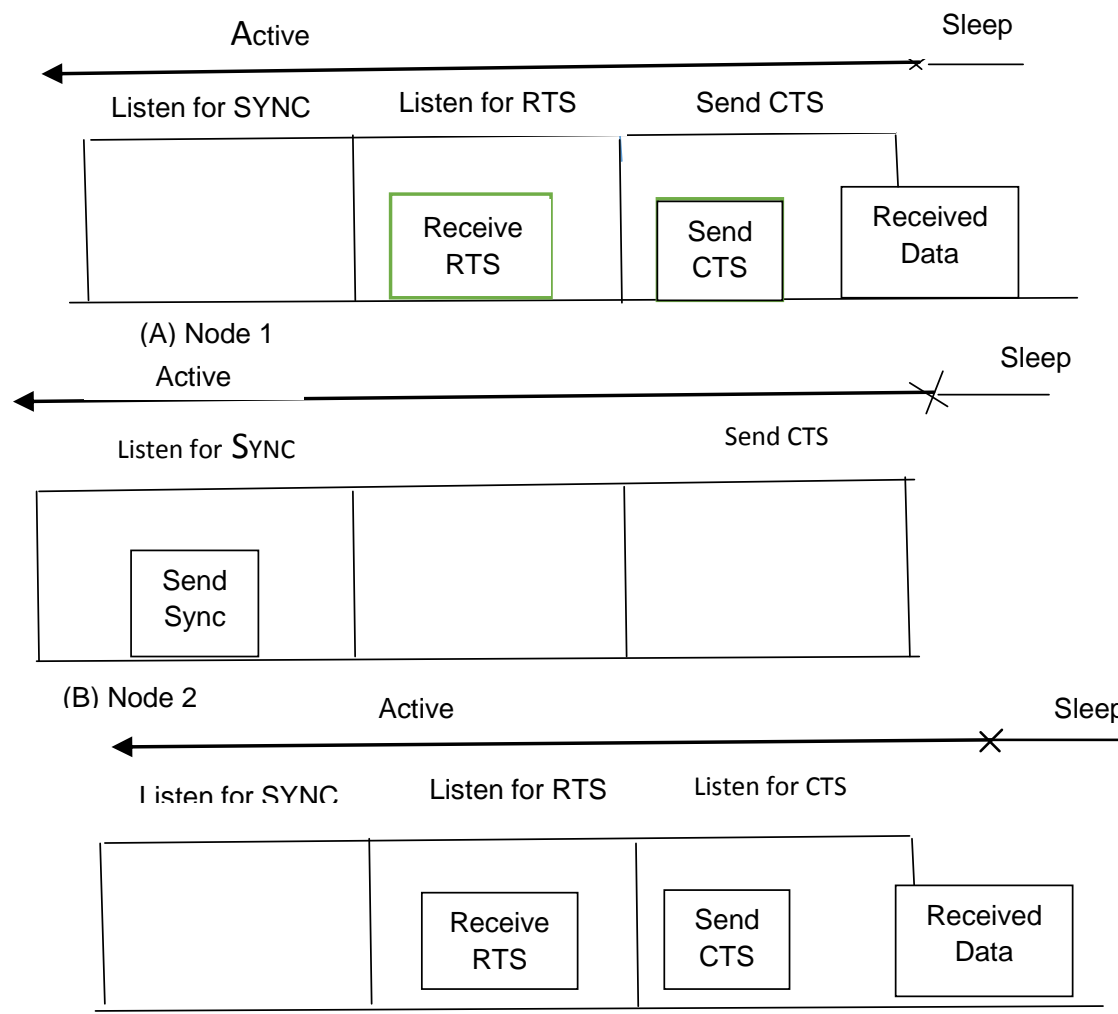

(C) Node 3

Fig. 1 SENSOR MAC Protocol [8]

With the low-duty-cycle operation, nodes must delay sending a packet until the next listen period of a destination, which increases latency.

\section{ALGORITHM}

1. If node i's RTS backoff timer expires and node i's RTS status is not off

2. If node $i$ is located in the observation circle in the center of the given area

Increase the number of RTS sent by 1

3. Check every neighbouring node $j$ of node $i$

If node j's RTS expires within 2 time slots

Set node i's RTS status as collision

End If

Set node j's RTS status as collision

If node j's CTS expires within 2 time slots

Set node i's RTS status as collision

End If

Set node j's CTS status as collision

If node j's RTS expires after 2 time slots

End If

Set node j's RTS status as off

4. If node i's RTS status if not collision

Set node i's RTS status as sent

Cancel the RTS backoff at node i's destination

Set CTS backoff time at node i's destination as

current time slot +2

\subsection{Power Efficient Gathering in Sensor Information Systems (PEGASIS)}

The main idea in PEGASIS [22,9] is for each node to receive from and transmit to close neighbours and take turns being the leader for transmission to the BS. This approach will distribute the energy load evenly among the sensor nodes in the network. We initially place the nodes randomly in the play field, and therefore, the i-th node is at a random location. The nodes will be organized to form a chain, which can either be accomplished by the sensor nodes themselves using a greedy algorithm starting from some node. To construct the chain [19], we start with the furthest node from the BS. We begin with this node in order to make sure that nodes farther from the BS have close neighbours, as in the greedy algorithm the neighbour distances will increase gradually since nodes already on the chain can be revisited. When a node dies, the chain is reconstructed, fig. 2 , in the same manner to bypass the dead node.

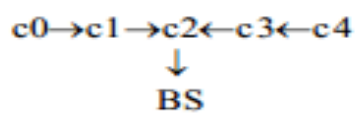

Fig. 2 Token Passing in PEGASIS [19]

PEGASIS performs data fusion at every node except the end nodes in the chain. Each node will fuse its neighbour's data with its own to generate a single packet of the same length and then transmit that to its other neighbours. In the above example, node $\mathrm{c} 0$ will pass its data to node $\mathrm{c} 1$. Node $\mathrm{c} 1$ fuses node c0's data with its own and then transmits to the leader. After node $\mathrm{c} 2$ passes the token to node $\mathrm{c} 4$, node $\mathrm{c} 4$ transmits its data to node $\mathrm{c} 3$. Node c3 fuses node $\mathrm{c} 4$ 's then transmits to the leader. Node c2 waits to receive data from both neighbours, fuses its data with its neighbour's data and finally transmits one message to the BS. 


\section{ALGORITHM}

1. Define the Initial and Destination Node called Si and Di

2. Find the MinDistance and MinDistance Path between Si and $D i$

3. Find All N Neighbor Nodes of Si called Neighbouri $<$ DistanceVector

4. For $I=1$ to $N$

Find MinDistance,MinEnergy,Cidel for all the Neighbours nodes

If $($ Energy $($ Neighbour $))=$ MinEnergy \&\&

Cidel(Neighbour) $)<=$ Threshold \&\&

Distance $($ Neighbour $)=$ MinDistance

\section{SIMULATION SETUP}

Both the MAC protocols SENSOR MAC and IEEE 802.11 based PEGASIS are good in their place but they cannot be used together so here will be comparing both the protocols on the basis of throughput, end to end delay and the amount of packets received and the packets lost, thus evaluating the correct use of protocol at right places such as either for small area sensor network or for large localities area. For analyzing, the algorithms examined the evaluation on NS2 simulator with various parameters describe as in the table 1.

Table I Parameters Used for Simulation

\begin{tabular}{|l|l|}
\hline DESCRIPTION & PARAMETER \\
\hline Area of Deployment & $670 \mathrm{~m} \times 670 \mathrm{~m}$ \\
\hline Traffic type & CBR \\
\hline Channel type & Wireless/Phy \\
\hline MAC layer & 802.11, SENSOR MAC \\
\hline No. of nodes & $3,10,20$ \\
\hline Propagation model & Two ray ground \\
\hline Queue length & 50 \\
\hline Interface queue type & DropTail/PriQueue \\
\hline Antenna & Omni Antenna \\
\hline Simulation Time & 200sec \\
& \\
\hline
\end{tabular}

Set Neighbour node as Next node

Else if Distance $($ Neighbour $)=$ MinDistance

Set Neighbour node as Next node

Else $($ Energy $($ Neighbour $))=$ MinEnergy

Set Neighbour node as Next node

Else if $($ Energy $($ Neighbour $))=$ MinEnergy and

Distance $($ Neighbour $)=$ MinDistance

Set Neighbour node as Next node

\section{RESULT ANALYSIS}

As already outlined in this paper taken two MAC protocols, namely SENSOR MAC and PEGASIS. The mobility model used is Random waypoint mobility model because it models the random movement of the mobile nodes. For correctness of result here AWK have used and following simulations outputs comes to study and analyses the result .These are NS2 network simulator, NAM editor to show the animated schema of the two protocols under study viz. SENSOR MAC \& PEGASIS, their performances and their routing paths. Furthermore here represent used X-graph to graphically represent of the throughput [fig 3, fig 4 and fig 5], for both the protocols on different nodes values and hence comparing them. From the comparison the declaration for both the protocol as a SENSOR MAC \& PEGASIS that which is the capable of handling the routing process smoothly.

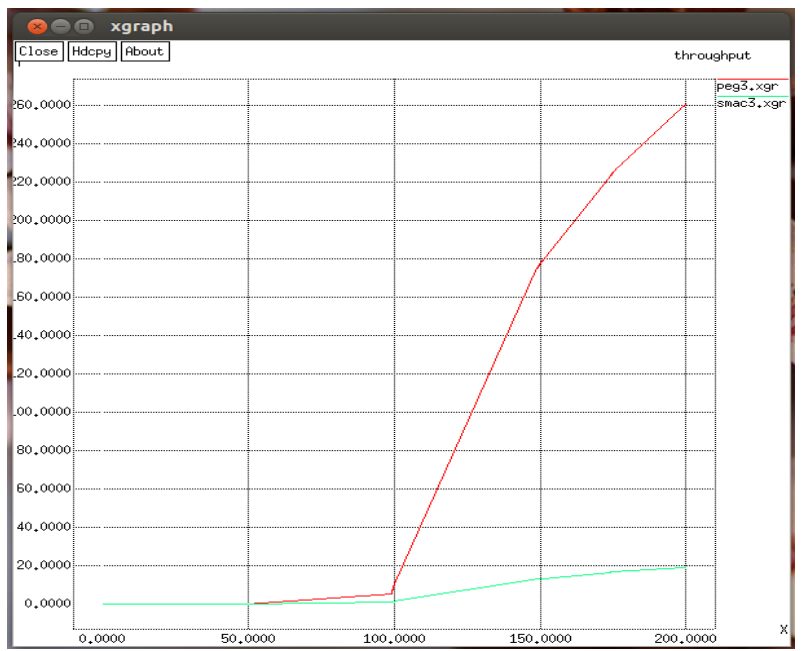

Fig.3 Xgraph for throughput comparison of PEGASIS and SENSOR MAC with 30 nodes

All the graph and outputs are achieved by using the AWK trace file method, the fig 3 and 4, show the Xgraph, in which pegasis throughput is higher than the s-mac, for small area coverage

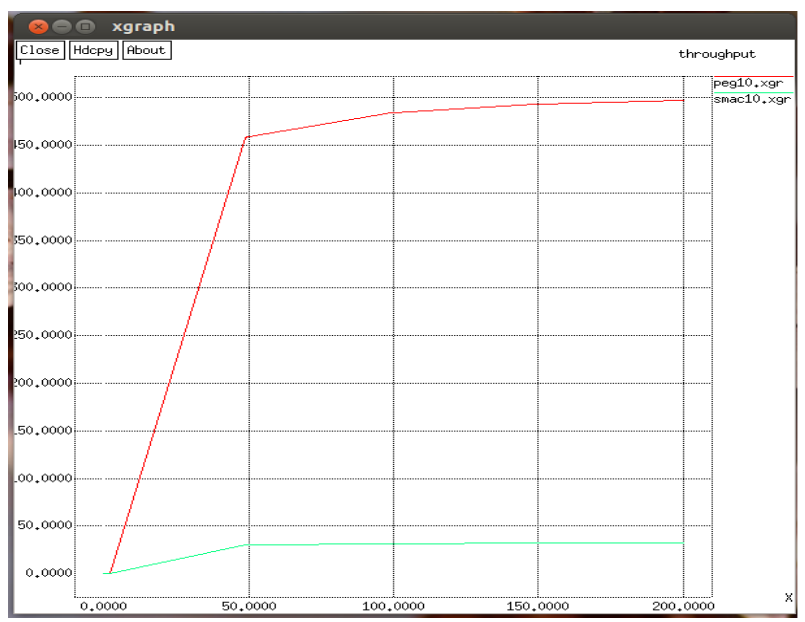

Fig.4 Xgraph for throughput comparison of PEGASIS and SENSOR MAC with 100 nodes 


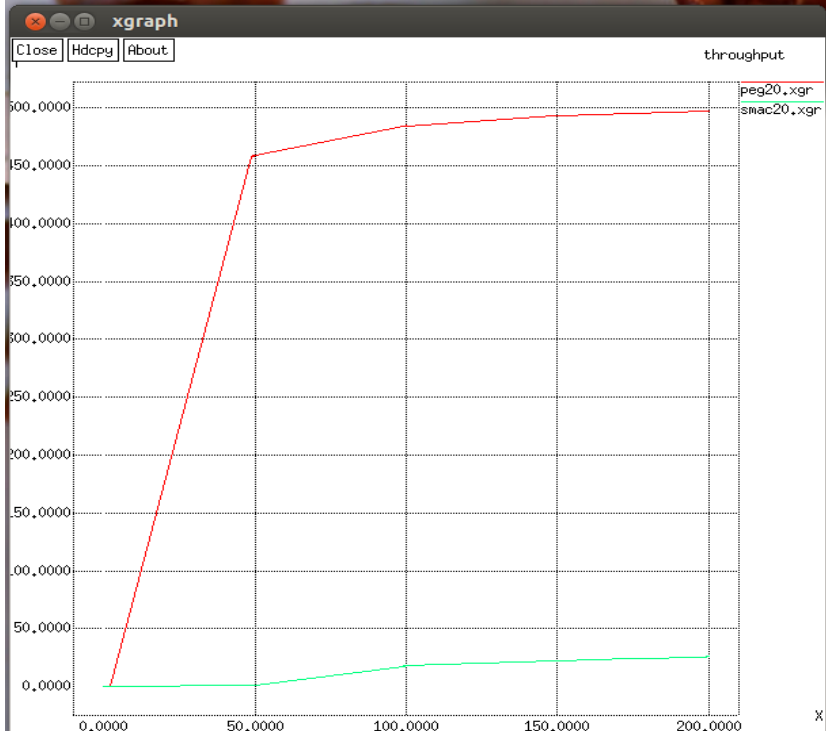

Fig.5 Xgraph for throughput comparison of PEGASIS and SENSOR MAC with 200 nodes

Finally end to end delay, average throughput and packet dropped and delay all these parameter we compared and the result are as fig 6 and Fig 7 for small area sensor network in which PEGASIS output is better than the S-MAC.

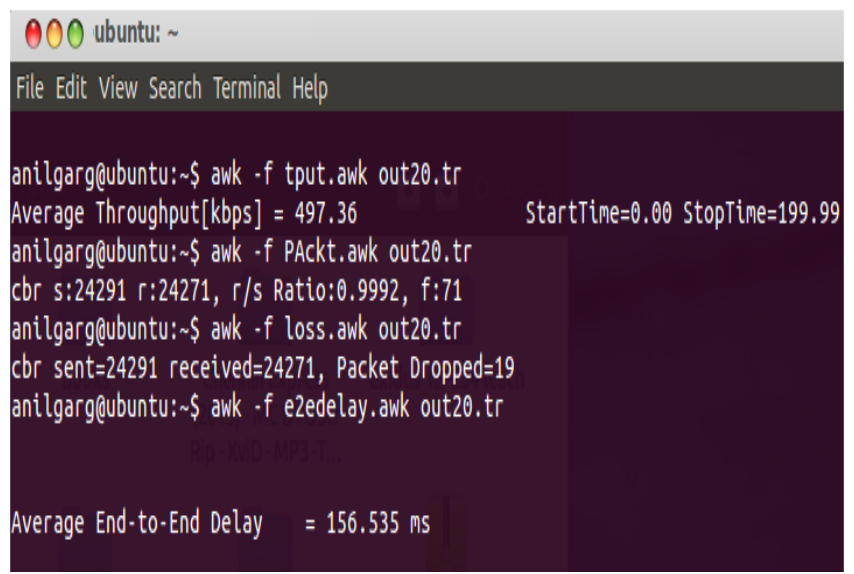

\section{Fig. 6 Output of End to End Delay for PEGASIS in ns2 terminal}

The above Figures $6 \&$ Fig 7, shows the Output of End-to-End Delay, Throughput .Packet Delivery Ratio and Packet Loss for SENSOR MAC and PEGASIS in ns2 terminal for 20 nodes.

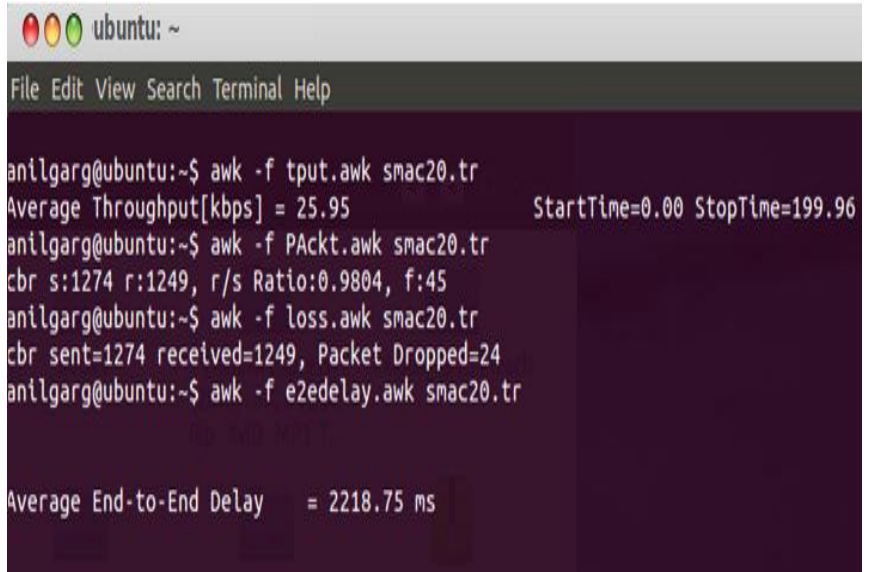

\section{Fig. 7 Output of End to End Delay for SENSOR MAC in ns2 terminal}

\section{CONCLUSION AND FUTURE WORK}

This paper compared the performance impact of sleep in a Sensor-MAC protocol and non-sleeping based IEEE 802.11 by evaluating PEGASIS, by analysis and simulation. The results demonstrate that the scenario that makes use of PEGASIS protocol exhibits higher throughput as compared to the scenario that uses SENSOR MAC protocol in small network area. This happens because in SENSOR MAC protocol the nodes undergo periodic sleep states to save energy. When the node is in sleep state then the other node has to wait for the node to enter listen mode so that data can be transferred. This waiting time reduces the throughput of the network. Also it is observed that the throughput increases as the no. of nodes increases, attains a highest value and then it starts decreasing as the no. of nodes further go up. As future work, there will be implemented on performance impacts of latency and energy consumption under varying duty cycles and for different packet arrival rates, etc.

\section{REFERENCES}

[1] Abdul Gani Khan, "A Comparative Analysis: Routing Protocols for Wireless Sensor Networks", Volume 2, International Journal of Research in IT \& Management (IJRIM), Issue 2, February 2012.

[2] Ou Yang, Wendi Heinzelman, "Modeling and Performance Analysis for SENSOR MAC with Retransmissions in Multihop Wireless Sensor Networks", IEEE, 2010.

[3] W. Heinzelman, A. Chandrakasan, and H. Balakrishnan. Energy-Efficient Communication Protocol for Wireless Microsensor Networks. In Proceedings of the Hawaii Conference on System Sciences, Jan. 2000.

[4] Kazem Sohraby, Daniel Minoli, Taieb Znati, "Wireless Sensor Networks- Technology, Protocols, and Applications", John Wiley \& Sons, Inc. Publication, 2010.

[5] Mostafa Ghannad, Vahid and Mehdi, "Critical Area Attention in Traffic Aware Dynamic Node Scheduling for Low Power Sensor Networks “, IEEE Communications Society / WCNC, 2005

[6] Wei Ye, John Heidemann, Deborah Estrin "An EnergyEfficient MAC Protocol for Wireless Sensor Networks", 2002.

[7] Wenjing Guo, Wei Zhang, Gang Lu,Department of Computer Science and Technology ,East China Normal 
University "PEGASIS Protocol in Wireless Sensor Network Based on an Improved Ant Colony Algorithm" IEEE-2010 Second International Workshop on Education Technology and Computer Science.

[8] T. S. Rappaport. Wireless Communications. PrenticeHall, 1996.

[9] Stephanie Lindsey, Cauligi S. Raghavendra, "PEGASIS: Power-Efficient Gathering in Sensor Information Systems", Computer Systems Research Department, 2000

[10] Wei Ye, J. Heidemann, and D. Estrin, "Medium access control with Coordinated, adaptive sleeping for wireless sensor networks," Technical Report ISI-TR-567, USC/Information Sciences Institute, January 2003.

[11] R. Steele. Mobile Radio Communications. Pentech Press, London, 1992.

[12] Pooja Singh, Vikas Pareekh \& Anil km Ahlawat "A review of Data Fusion in WSN Routing" in CiiT International Wireless Communication Journal May'13.Paper DOI: WC052013005.

[13] Demirkol I, Ersoy C, Alagoz F, (2006) "MAC Protocols for Wireless Sensor Networks: a Survey", IEEE Communications Magazine.

[14] Langendoen K, (2008) "Medium Access Control in Wireless Sensor Networks", Book Chapter in Medium Access Control in Wireless Networks, Volume II: Practice and Standards, Nova Science Publishers.

[15] Shwe, H.Y., Xiao-hong, J., Horiguchi, S, (2009) “ Energy Saving in Wireless Sensor Network", Journal of Communication and Computer, Vol. 6, No.5, pp. 2028.

[16] Study of Data fusion in Wireless Sensor Network Kiran Maraiya, Kamal Kant, Nitin Gupta, Proc. of the International Conference on Advanced Computing and Communication Tech. (ACCT 2011) Copyright () 2011 RG Education Society ISBN: 978-981-08-7932-7,Pg 535- 539.

[17] Yebin Chen, Data Fusion in Wireless Sensor Networks, Electronic Commerce and Security, 2009. ISECS '09. Second International Symposium, Volume: 2, Page(s): $504-509$.
[18] Y. Jeong, S. Lakshmanan, S. Kakumanu, and R. Sivakumar, "Cue-based Networking using Wireless Sensor Networks: A Video-over-IP Application," IEEE Comm, Society Conf. on Sensor, Mesh and Ad hoc Comm. \& Networks (SECON), San Francisco, CA, June 16-20, 2008.

[19] Stephanie Lindsey and Cauligi S. Raghavendra, "PEGASIS: Power-Efficient GAthering in Sensor Information Systems", Aerospace Corporation, Los Angeles.

[20] Hong Luo, Huixiang Tao, Huadong Ma, Sajal K. Das, Data Fusion with Desired Reliability in Wireless Sensor Networks, March 2011 (vol. 22 no. 3),pp. 501-513.

[21] Exploiting Data Fusion to Improve the Coverage of Wireless Sensor Networks Networking, IEEE/ACM Transactions on, April 2012 Author(s): Rui Tan ; Benyuan Liu ; Jianping Wang ; Xiaohua Jia Volume: 20, Issue: 2 Page(s): 450 - 462

[22] Sunita Rani, Tarun Gulati," An Improved PEGASIS Protocol to Enhance Energy Utilization in Wireless Sensor Network" International Journal of Advanced Research in Computer Science and Software Engineering; Volume 2, Issue 11, November 2012, ISSN: $2277128 \mathrm{X}$

[23] Dharma Prakash Agrawal, Ad- Hoc and Sensor Networks, Theory and applications, "carlos de morais cordeiro", Philips research North America, USA.

[24] The analysis of data fusion energy consumption in WSN, Author(s): Li Li, System Science, Engineering Design and Manufacturing Informatization (ICSEM), IEEE 2011 International Conference on 22-23 Oct. 2011, Volume: 1, Page(s): 310 - 313.

[25] K. V. Kale, S. C. Mehrotra, K.V. Kale, S.C. Mehrotra, R.R. Manza et al. "efficient MAC protocol for wsn" Computer Vision and Information Technology: Advances and Applications" csd, trans- 2012/06,

[26] Tarana Shaikh, Anand Mane et al. "MAC Protocols researched for Wireless Sensor Networks" ijcttjournal, Volume 4,issue-5,IJCTT-V4I5P100. 